\title{
Pivotal "New Normal" Telemedicine: secured psychiatric homeopathy medicine transmission in Post-COVID
}

\author{
Joydeep Dey ${ }^{1}$
}

Received: 5 September 2020/Accepted: 15 April 2021/Published online: 27 April 2021

(C) Bharati Vidyapeeth's Institute of Computer Applications and Management 2021

\begin{abstract}
In this Post-COVID-19 "New Normal" era, the importance of Telemedicine is immense. In telemedicine, the patients' sensitive medical data security is the matter of highest demands. There exists data transmission between the psychiatric patients and psychiatrists through webbased portal. Cryptography is the essential component where medical data is transmitted securely. Insomnia, Depression, Mood swings, Fear of death, etc are the common mental conditions observed in this COVID-19 pandemic. A symmetric key based on randomization of numbers were generated by a reliable and third party Key Distribution Centre. A set of robust binary sequences of 128 bits long were generated, known as secret session keys, and will be transported to both the parties. The web-portal will capture all the patients' symptoms. Then homeopathy E-Prescriptions would be generated by the psychiatrists. This transmission of E-Prescription would be done using the proposed secret session key using Advanced Encryption Standards. Ignatia, Natrum Sulphuricum, Aconite, Arsenicum Album, Belladonna, etc are the important homeopathy medicines as prescribed by the homeopathy psychiatrists in COVID-19. Mathematical calculations were done on this proposed technique with positive efficacy. Hence, homeopathy telemedicine is the pivotal way to cure psychiatric patients remotely in this Post-COVID19 "New Normal" context.
\end{abstract}

Joydeep Dey

joydeepmcabu@gmail.com

1 Department of Computer Science, State Aided College Teacher and HoD, M. U. C. Women's College, Burdwan, WB, India
Keywords Telemedicine - Psychiatric diseases · Homeopathy E-prescription · Encryption time

\section{Introduction}

In this COVID-19 pandemic, most of us are really frustrated due to coronavirus [1]. Most of the people are suffering from some sorts of metal disorders. Mental disorder is the illness and unstable frame of mind that leads to unhappy, unsatisfied, and non-productive life. Alzheimer's disease, Schizophrenia, dementia, mood fluctuation, insomnia, depression, behavioral disorder, etc are the most frequent mental disorders observed during this global COVID-19 pandemic. In this COVID-19 era, most of the people are suffering from some sort of psychiatric problems. The major causes behind these mental disorders are anxiety, stress, unhealthy life style, low diets, genetics, hormonal imbalance, etc. There exists so many problems likes of frustration in working place, deadlines of works, pressure, stress, exhaustiveness, failure in projects, breakdown in jobs, etc. The impact of this coronavirus is so immense that led to rapid surge in the telemedicine services. In the psychiatric fields, majority of the patients are not willing to visit the psychiatrists due to several factors. Shyness and inferiority feelings of the psychiatric patients are to be sorted out here. This problem has been addressed in this proposed technique. A transmission guided through online web-portal for the homeopathic psychiatric medicines has been proposed here. Patients' medical data security has been kept under secured scheme using data encryption. Telehealth system is an efficient mechanism to cure the patients in this global COVID-19 era [2]. Cryptography is the science to ensure data security from unwanted access to the sensitive data and information [3]. 
The objective here is to treat the psychiatric patients using web-portal E-health system. The proposed system works on the secret symmetric key cryptography. The same key is used at the time of encryption by the psychiatrists, and also at the time of decryption by the patients. The same secret key has been obtained through some of the authenticated key distribution centers (KDC). Before the transmission of the homeopathy psychiatric medicines, the secret key has been transferred to both the parties through protected channel. To counterfeit the Man-In-The-Middle attacks, the proposed approach of generating keys is a suitable technique in such E-Health systems. Online telehealth services are far better in this pandemic situation. Patients can take medicines, consult to their doctors, health checkups, etc. Most importantly, these services are taken from remote locations too. No chances of noscomial infections for the patients. Travelling cost and getting affected by the corona virus is being cancelled.

Serotonin is a hormone which is produced by the human brain. Its enough secretion results in better mood, and deficit results in depression. Psychiatric diseases are also contributed through inheritance. The positive thing of this paper is that psychiatric patients can avail their homeopathic treatments from their homes. The transmission of E-medicines is the main objective of this proposed technique. Homeopathy is based on the philosophy of curing the patients' mental and physical health overall. Health is a matched frame of harmony dwelling with mental peace and physical fitness. Homeopathy treatment is an alternative medicine that persists with enormous critiques on its scientific approach. Homeopathy medicines are selected based on patients' existing conditions and then potency of the dose is selected. Following are the existing conditions which imparts in determining the homeopathy medicines. They are fear, anxiety, sleep, attitude, stress, dreams, OCD, sense of humor, reactions against common instances, etc. Some common medicines used in homeopathy treatments are listed as follows [4].

Calcarea Carbonica and Aconite are given for anxiety with palpitations, dry mouth, etc. Ignatia is used for grief, loss, mood changer, etc. Kali phophoricum is prescribed for over whelming the wishes that are imaginary. Those patients take too much stress on themselves. Lycopodium is prescribed due to lack of self confidence. Sepia is given to menopause females with changing moods.

\section{Related works}

Torous et al. [5] had briefly explained the presence scenario of COVID-19 pandemic and its implications on the mental health. Also the surge of mobile health is emerging now. Narita et al. had shown that there exists a direct relationship between the loneliness and mental complications. They had measure Psychotic Experience (PE) on the US populations under adjustment of confounding factors. Then regression models were used to show the linkage between the PE and loneliness [6]. Whaibeh et al. said that due to unprecedented pandemic there has been a crisis in the systematic psychiatric research. There should be more mental health care systems to treat the mentally challenged patients [7]. Torales et al. had reviewed the articles covering mental health in this global COVID-19 pandemic. They had compared the mental health status as compared to other earlier severe global infections. According to them this COVID-19 had caused more mental complications like stress, anxiety, denial, acute depression, insomnia, fear, anger, etc. More than that the co-morbity adds more psychological issues to the patients [8]. Kavanagh et al. said that telemedicine has become an emergent component in South Australia. They had reviewed the pros and cons of telepsychiatry in this paper [9]. Bao Y. et al. [10] had strived to improve the mental awareness in this pandemic situation. They have stated that psychiatrists and psychologists are providing online mental support to remain fit in this COVID-19 pandemic situation in China. This would help the mental health of the general population. Cao et al. [11] had survey medical students on cluster sampling technique. They have used GAD-7 scale points to evaluate the mental health of college students studying medical science. The mental health of a student is important during the time of public emergencies. Cui et al. had written about different challenges that are faced in psychiatric treatments in this COVID-19 era [12]. Patients are facing huge problems by not having treatments properly. Freeman [13] had stated that the anticipation of the corona virus cannot be done now. There should be new set up in the telemedicine sectors to speed up the treatments from remote places. Psychiatric treatments on several mental disorders may be possible through telemedicine. Kang et al. [14] said that psychiatrists can perform an integral role in the mental happiness of the COVID-19 patients and other health care givers. The psychological issues must be addressed in this pandemic situation too.

Algarni et al. [15] had proposed an ECG based cryptosystem which is used in telemedicine. High speech masking signals were used to fuse the original ECG signals. They had compared their technique with the encryption technique which uses one dimensional logistic map, its performance was measured through statistical tests such as histogram, structural similarity index, Signal-to-Noise Ratio (SNR), correlation coefficient, etc. Murillo-Escobar et al. [16] had proposed an encryption algorithm based on double layer chaotic function. Clinical signals were used to encrypt in the field of telemedicine. Tamilarasi et al. [17] had proposed a novel hybrid lightweight encryption 
method on EEG signals based on swarm optimization algorithm. They had optimized the key space using their proposed algorithm. The performance metrics were measured to evaluate their method. Thanki et al. [18] had proposed a robust and hybrid watermarking technique for providing medical image security in telemedicine. Experimental results in their technique had shown high imperceptibility and robustness against different types of data attacks. Moorthi et al. [19] had reviewed the research papers related to COVID-19 cases in China. Based on the symptoms and case history, they had suggested their psychiatric homeopathy medicines to treat such patients. Arsenic album, Calcarea carbonica, Ignatia, Lachesis, Pulsatilla had obtained the highest ranks in their analysis.

\section{Existing problems}

Due to following problems, the psychiatric patients are not interested to visit the physician's chambers in this COVID19 pandemic, and then their problems get unsorted. The problems are based on their mental rigidity. Fear of social popularization is one of the main problems faced by those psychiatric patients. Stigmatized of being COVID-19 positive is another factor by the society. Non-participation and

\section{Proposed technique}

The proposed web-based portal will be able to treat the patients by abiding the COVID-19 protocols. Since there are restrictions on external works, so psychiatric patients can be treated through this methodology. Moreover, the ashamed feelings of consulting the psychiatrist personally are being avoided by this. The patient wants to communicate with the psychiatrist by this web-based portal, and then the Key Distribution Center (KDC) will generate a secret session key. This secret key would be communicated to both the patient and psychiatrist through the protected channel. Randomized emphasis has been given on the generation of 128 bits long secret session key by the KDC. Through online portal, different symptoms would be stored into the server. Different parameters that need to be accumulated from the patients' end are age, sex, periodical status (females only), mental conditions, diabetic status, blood pressure, etc. The homeopathy physician would analyze those collected symptoms and then prescribe homeopathic medicines by the E-Prescriptions. The proposed technique serves the transmission of this E-Prescription in a secured way. A key distribution centre will use random functions [11-13] to diffuse the hackers.

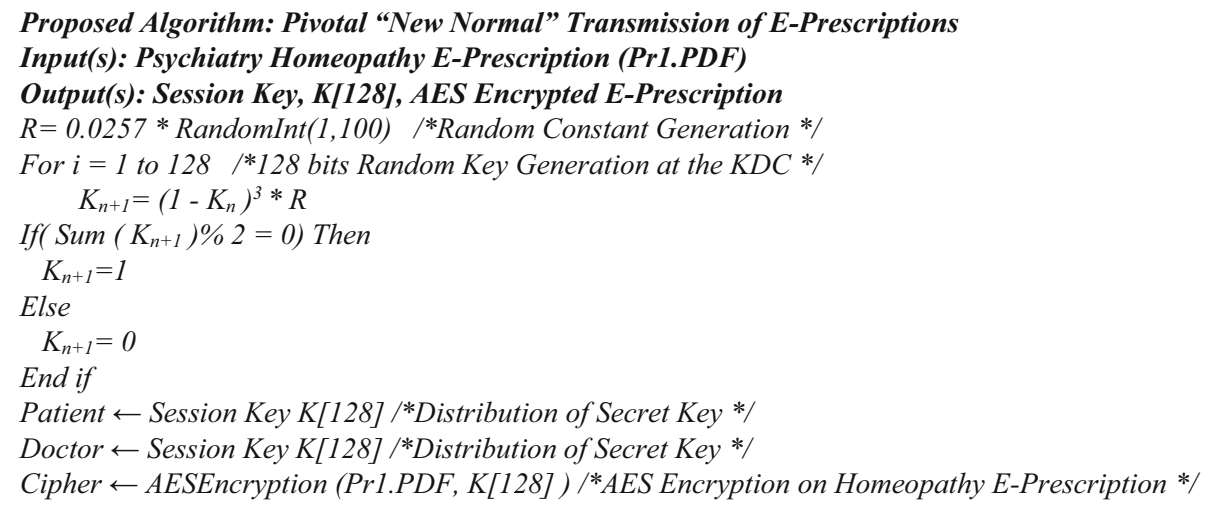

non-cooperation by these patients while treatments under progress, is another issue here. Physical consultations may lead to the COVID-19 attacks quickly.

Also there lies low data protection during the public channel transmission in case of telehealth systems. Eavesdropping on the patients' data is the common form of attacks in such digital health platforms. The secret keys used in the process of transmission are likely to be compromised by the intruders.
To treat the psychiatric patients in this COVID-19 era is the main objectivity here as shown in the above algorithm. A binary sequence of 128 bits was generated by the third party i.e. Key Distribution Center. Since the patients' data are to be preserved against different malicious attacks. AES encryption is a classical and effective algorithm that has been used here. Thus, the proposed technique will give a new dimension in the transmission of E-Prescriptions.

In the field of online telemedicine especially in this post COVID-19 situation, patients' data are to be kept secret. The novelty of this proposed technique is that a third party i.e. Key Distribution Center (KDC) has generated a session 
Table 1 Set of proposed session keys

Table 2 Encryption and decryption time needed on key 1

Fig. 1 Histogram of offline E-prescription

Fig. 2 Autocorrelation of offline E-prescription

\begin{tabular}{lll}
\hline Session key Id & $\begin{array}{l}\text { Session key } \\
\text { (given in Hexadecimal code) }\end{array}$ & $\begin{array}{l}\text { Session number } \\
\text { (where secret key used) }\end{array}$ \\
\hline 1 & 078DBF79285F9D50695ED4DDBDE1564D & Session 6 \\
2 & 1095121044A82B966057D32AB082DA56 & Session 4 \\
3 & 3645E6F3D378EF2C1C768BC6813C42D5 & Session 2 \\
4 & 746D54BBE308B5FCD4B9F05E2536A506 & Session 10 \\
5 & EBF8C8AA4351457353FE83967AC3495B & Session 7 \\
6 & AB6A8CA27CA95036F3EDA8AD49EC5A69 & Session 1 \\
7 & 5886F444AB623E10B55BB1398925F7A8 & Session 5 \\
8 & F5238976B37F0EBD5D1D826F4B2C217E & Session 9 \\
9 & 3E48BF11DACDDF353350B4E27CA7478F & Session 3 \\
10 & B6BB014A666BF9F47E17C163A9892EFB & Session 8 \\
\hline
\end{tabular}

\begin{tabular}{llllll}
\hline $\begin{array}{l}\text { Sl } \\
\text { no }\end{array}$ & $\begin{array}{l}\text { Size of } \\
\text { E-prescription }\end{array}$ & $\begin{array}{l}\text { Used session } \\
\text { key }\end{array}$ & Encryption time & Decryption time & Total time \\
\hline 1 & $3.15 \mathrm{MB}$ & $078 d b f 79285$ & $2575 \mathrm{~ms}$ & $1896 \mathrm{~ms}$ & $4444 \mathrm{~ms}$ \\
2 & $2.64 \mathrm{MB}$ & $f 9 d 50695 e d 4$ & $2156 \mathrm{~ms}$ & $1765 \mathrm{~ms}$ & $3921 \mathrm{~ms}$ \\
3 & $1.78 \mathrm{MB}$ & $d d b d e 1564 d$ & $1845 \mathrm{~ms}$ & $1540 \mathrm{~ms}$ & $3385 \mathrm{~ms}$ \\
4 & $2.85 \mathrm{MB}$ & & $1544 \mathrm{~ms}$ & $1600 \mathrm{~ms}$ & $3144 \mathrm{~ms}$ \\
5 & $5.16 \mathrm{MB}$ & & $3183 \mathrm{~ms}$ & $2897 \mathrm{~ms}$ & $6080 \mathrm{~ms}$ \\
\hline
\end{tabular}
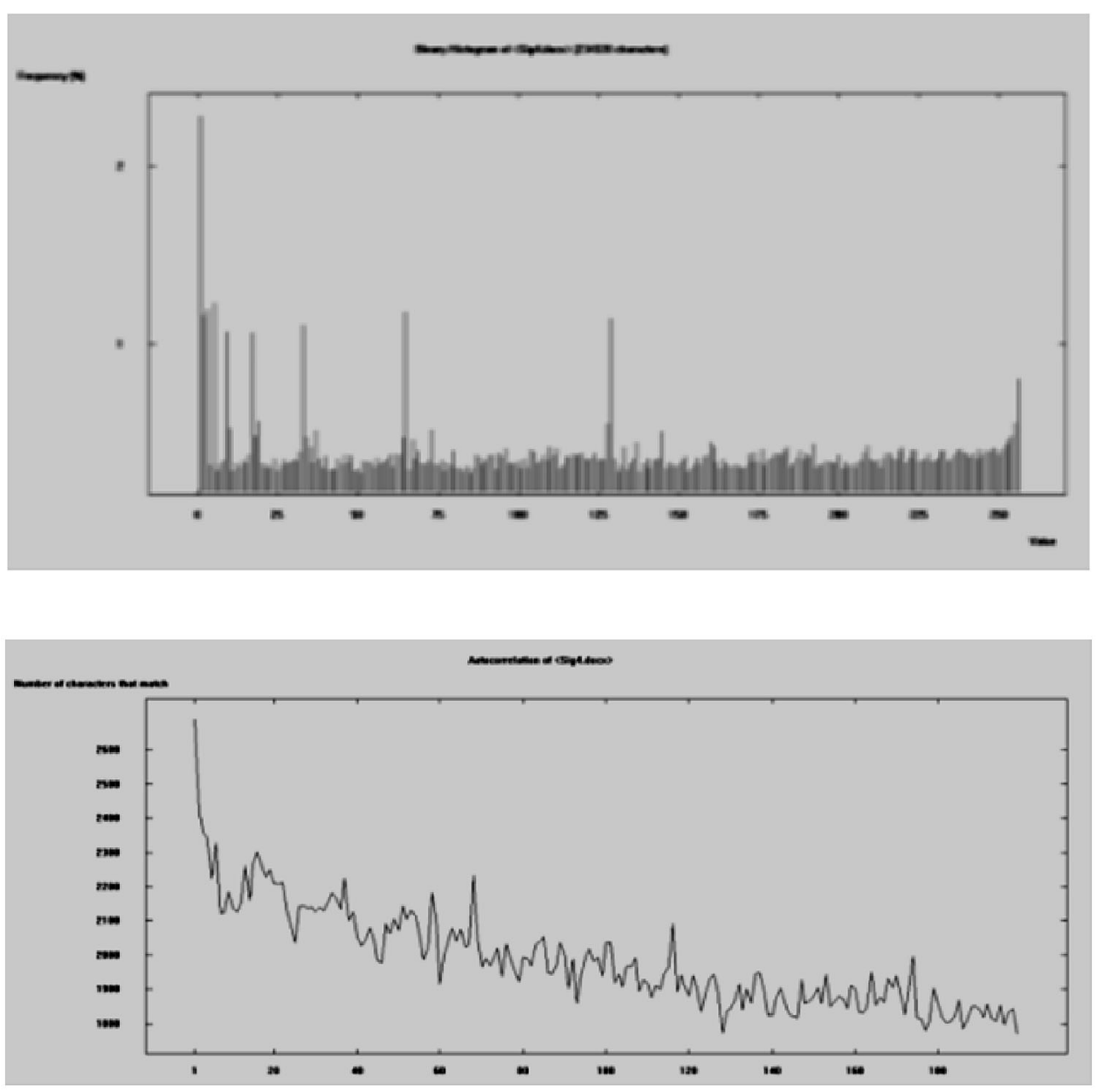
Fig. 3 Histogram diagram and autocorrelation diagram of AES encrypted E-prescription

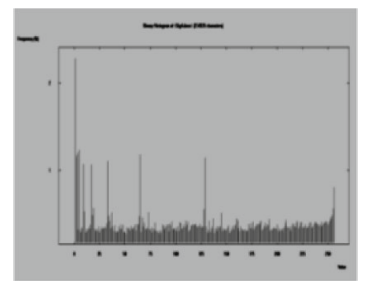

Histogram of AES Encrypted E-Prescription

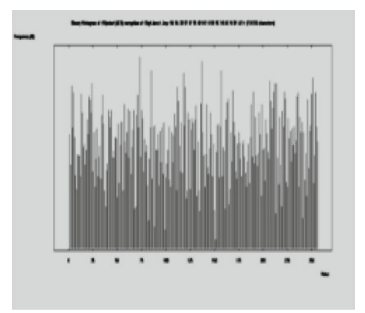

Histogram of AES Encrypted E-Prescription

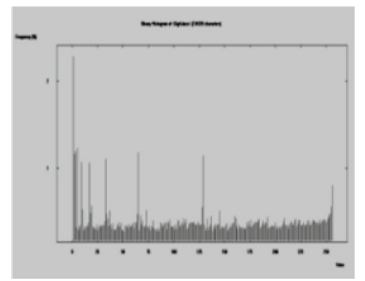

Histogram of AES Encrypted E-Prescription

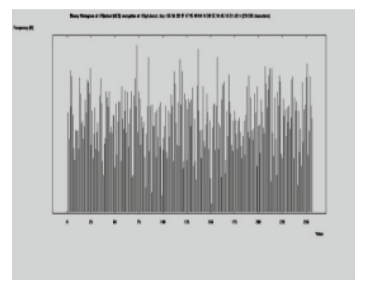

Histogram of AES Encrypted E-Prescription

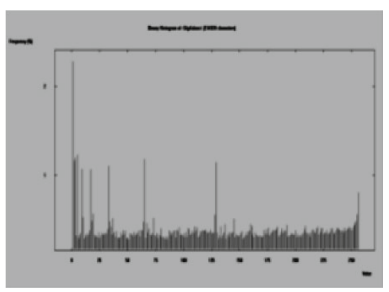

Histogram of AES Encrypted E-Prescription
Session 5

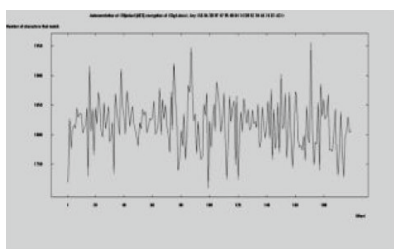

Autocorrelation of AES Encrypted E-Prescription

Session 9

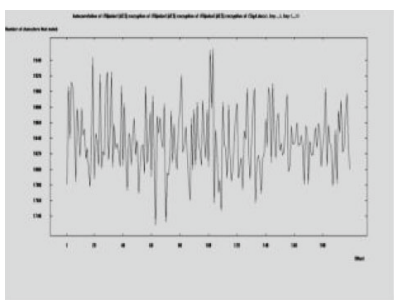

Autocorrelation of AES Encrypted E-Prescription

Session 3

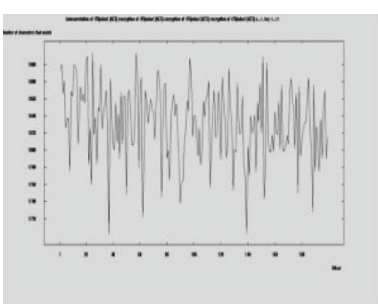

Autocorrelation of AES Encrypted E-Prescription

Session 7

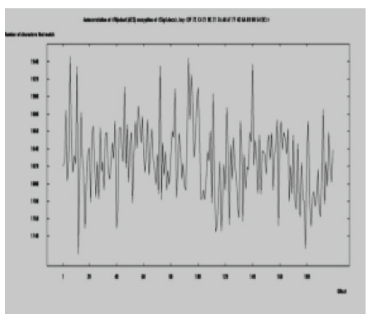

Autocorrelation of AES Encrypted E-Prescription

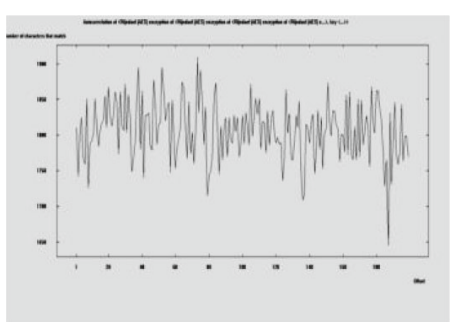

Autocorrelation of AES Encrypted E-Prescription 
key, and which is being used as encryption and decryption process. Thus, the problem of compromised key generation algorithm has been reduced. AES encryption [20, 21] is used to encrypt the homeopathic prescriptions. Moreover, in this paper, session of 128 bits has been generated by the $\mathrm{KDC}$ i.e. key size $\mathrm{Sk}=128$. The length of the session key has been denoted as $\mathrm{Lk}$, i.e. $\mathrm{Lk}=4$ with 32 bits per column. The number of rounds present in this AES of the proposed technique is denoted by $\mathrm{Rn}$ i.e. $\mathrm{Rn}=10$. Through this proposed methodology, loads of psychiatric patients can be treated remotely in this COVID-19 pandemic. This system is more immune and robust against the malicious attacks inside the telemedicine networks.

\section{Results}

A secret key has been generated by the reliable thirds party Key Distribution Centre. The proposed key generation can be illustrated briefly as in the following. A random number (R) has been generated of any length. Let R gets 1902 as randomized value. Then sum of its digits will be $12(1+9+0+2=12)$. When 12 is modularly divided by 2 , then 0 is obtained. So this ' 0 ' will be filled at $0^{\text {th }}$ index of Session Key. Similarly, 128 epochs will be done to have Session Key of 128 bits long.

As defined above, the following Table 1 contains the set of 10 secret keys. All keys are randomly chosen for different transmission sessions. Thus, intruders will not be able to know the correct session key.

In the above stated Table 1, a set of 128 bits keys were generated by the proposed technique at the Key Distribution Centre (KDC). The proposed key generation technique is much more robust because there exists no correlation between any two pair of secret keys. Encryption of the E-Prescription by the AES algorithm [22] any above stated keys will make the intruders' task much difficult. Patients' medical data [23] shall remain under confidentiality in this approach $[24,25]$. Through such proposed through online web-based portals will treat many psychiatric patients in this era of Post-COVID-19.

Different Homeopathy E-Prescriptions of varying size were taken into consideration under study. The secret keys as mentioned in the above Table 1 are used one by one for encryption and decryption method. The following Table 2, it shows the summary of those parameters as obtained when tested.

From histogram and auto correlation study, the following Figs. 1 and 2 indicate the histogram and autocorrelation of the traditional offline prescription respectively. There exists huge amount of non-uniformity at the distribution of character patterns. Autocorrelation curve is a scattered here in Fig. 3.
The histogram and autocorrelation graph analysis by the random secret keys given in Table 1 are done as in the following figure. The outcomes are the encrypted graphs using AES technique during various transmission sessions.

The intruders will not detect the secret session key as proposed in this techniqe. AES enabled ecnryption using the above stated keys (as mentioned in Table 1) for different randomly selected transmission sessions has provided good and satisfactory outcomes. Hence the proposed technique may be validated.

\section{Conclusion}

The results obtained in this proposed technique are goog enough to be an effective way to treat the mentally challenged patients in this Post-COVID-19 era. Homeopathy tratment is always an efficient medical treatment in this pandemic. The patients' data are kept well secured through this web-based treatment technique [25]. More importantly, this technique is more robust against the intrudding mechanism. Time required for encryption and decryption is within normal limits too. Thus, it provides a secure transmission on psychiatric homeopathy E-Prescription through web portal.

\section{Future scope of work and limitations}

The future scope of this paper is that an automated expert intelligent system may be developed without the involvements of any doctors. It may includes different specialized aspects of the medical sciences. It would be more effective in the field of telemedicine. The limitation of this proposed technique is that AES suffers from side channel attacks. Moreover, extra cost has been incurred interms of third party key generation and supply to both the users of the system through protected media.

Acknowledgement Author deeply acknowledges the medical support and inspiration received from Dr Salim Ahmad [B.H.M.S. Kolkata], Consultant Physician, Assistant Teacher in Nutrition, Vidyasagar Uchcha Vidyalaya, Purba Bardhaman, West Bengal, and Former Guest Lecturer, Department of Nutrition, M.U.C. Women's College, Burdwan, West Bengal.

\section{References}

1. Yamin M (2020) Counting the cost of COVID-19. Int $\mathrm{j}$ inf tecnol 12:311-317. https://doi.org/10.1007/s41870-020-00466-0

2. Sarkar A, Dey J, Chatterjee M, Bhowmik A, Karforma S (2019) Neural soft computing based secured transmission of intraoral gingivitis image in E-health. Indonesian J Electrical Eng Computer Sci 14(1):178-184 
3. Bhowmik A, Sarkar A, Karforma S, Dey J (2019) A symmetric key based secret data sharing scheme. Int J Computer Sci Eng 07(01):188-192

4. Viksveen P et al (2018) Homeopathy in the treatment of depression: a systematic review. Eur J Integ Med 22:22-36

5. Torous J, Keshavan MS (2020) COVID-19, mobile health and serious mental illness. Schizophr Res. https://doi.org/10.1016/j. schres.2020.04.013

6. Narita Z, Stickley A, DeVylder J (2020) Loneliness and psychotic experiences in a general population sample. Schizophr Res. https://doi.org/10.1016/j.schres.2020.01.018.pii:S09209964(20)30034-7

7. Whaibeh E, Mahmoud H, Naal H (2020) Telemental health in the context of a pandemic: the COVID-19 experience. Curr Treat Opt Psychiatry. https://doi.org/10.1007/s40501-020-00210-2

8. Torales J, O'Higgins M, Castaldelli-Maia JM, Ventriglio A (2020) The outbreak of COVID-19 coronavirus and its impact on global mental health. Int J Soc Psychiatry. https://doi.org/10. 1177/0020764020915212

9. Kavanagh S, Hawker F (2001) The fall and rise of the South Australian telepsychiatry network. J Telemed Telecare 7(Suppl 2):41-43

10. Bao Y, Sun Y, Meng S, Shi J (2020) Lu2019-nCoV epidemic: address mental health care to empower society. Lancet 395(10224):37-38

11. Cao Wenjun, Fang Ziwei, Hou Guoqiang, Han Mei, Xinrong Xu, Dong Jiaxin, Zheng Jianzhong (2020) The psychological impact of the COVID-19 epidemic on college students in China. Psychiatry Res. https://doi.org/10.1016/j.psychres.2020.112934

12. Cui LB, Wang XH, Wang HN (2020) Challenges facing coronavirus disease 2019: Psychiatric services for patients with mental disorders. Psychiatry Clin Neurosci. https://doi.org/10.1111/pen. 13003

13. Freeman MP (2020) COVID-19 From a Psychiatry Perspective: Meeting the Challenges. J Clin Psychiatry. https://doi.org/10. 4088/JCP.20ed13358

14. Kang C, Yang S, Yuan J, Xu L, Zhao X, Yang J (2020) Patients with chronic illness urgently need integrated physical and psychological care during the COVID-19 outbreak. Asian J Psychiatr. https://doi.org/10.1016/j.ajp.2020.102081

15. Algarni AD, Soliman NF, Abdallah HA et al (2021) Encryption of ECG signals for telemedicine applications. Multimed Tools
Appl 80:10679-10703. https://doi.org/10.1007/s11042-02009369-5

16. Murillo-Escobar M, Cardoza-Avendaño L, Lopez-Gutierrez R, Cruz-Hernández C (2017) A double chaotic layer encryption algorithm for clinical signals in telemedicine. J Med Syst. https:// doi.org/10.1007/s10916-017-0698-3

17. Tamilarasi K, Jawahar A (2020) Medical data security for healthcare applications using hybrid lightweight encryption and swarm optimization algorithm. Wireless Pers Commun 114:1865-1886

18. Thanki R, Kothari A (2020) Multi-level security of medical images based on encryption and watermarking for telemedicine applications. Multimed Tools Appl. https://doi.org/10.1007/ s11042-020-09941-z

19. Sanjeevi Karunakara Moorthi P, Radhika KC (2020) Muraleedharan, Psychological implications during the outbreak of COVID19 and its homoeopathic management. Indian J Res Homoeop 14(2):136-142

20. Arab A, Rostami MJ, Ghavami B (2019) An image encryption method based on chaos system and AES algorithm. J Supercomput 75:6663-6682. https://doi.org/10.1007/s11227-019-02878-7

21. Bashir A, Hasan AS, Almangush H (2012) A new image encryption approach using the integration of a shifting technique and the AES algorithm. Int J Comput Appl 975:8887

22. Kumar MT, Karthigaikumar P (2020) A novel method of improvement in advanced encryption standard algorithm with dynamic shift rows, sub byte and mixcolumn operations for the secure communication. Int J Inf Tecnol 12:825-830. https://doi. org/10.1007/s41870-020-00465-1

23. Bhowmik A, Dey J, Sarkar A, Karforma S (2019) Computational intelligence based lossless regeneration (CILR) of blocked gingivitis intraoral image transportation. IAES Int $\mathbf{J}$ Artif Intellig (IJAI) 8(3):197-204

24. Yamin M (2018) IT applications in healthcare management: a survey. Int J Inf Tecnol 10:503-509. https://doi.org/10.1007/ s41870-018-0203-3

25. Sarkar A, Dey J, Karforma S (2019) Secured session key-based E-health: biometric blended with salp swarm protocol in telecare Portals. In: Mandal J, Mukhopadhyay S (eds) Proceedings of the Global AI Congress 2019. Advances Intelligent Systems and Computing, vol 1112. Springer, Singapore 